\title{
A novel in vivo method to evaluate trueness of digital impressions
}

\author{
Emad A. Albdour ${ }^{1,2}$, Eman Shaheen ${ }^{1}$, Myrthel Vranckx¹, Francesco Guido Mangano ${ }^{3}$, Constantinus Politis ${ }^{1}$ \\ and Reinhilde Jacobs ${ }^{1,4^{*}}$ (D)
}

\begin{abstract}
Background: Intraoral scanners are devices for capturing digital impressions in dentistry. Until now, several in vitro studies have assessed the trueness of digital impressions, but in vivo studies are missing. Therefore, the purpose of this study was to introduce a new method to assess trueness of intraoral scanners and digital impressions in an in vivo clinical set-up.
\end{abstract}

Methods: A digital impression using an intraoral scanner (Trios ${ }^{\circledR}$ Cart wired, 3Shape, Copenhagen, Denmark) and a conventional alginate impression (Cavex Impressional ${ }^{\circledR}$, Cavex, Haarlem, the Netherlands) as clinical reference were made for two patients assigned for full mouth extraction. A total of 30 teeth were collected upon surgery after impressions making. The gypsum model created from conventional impression and extracted teeth were then scanned in a lab scanner (Activity 885 ${ }^{\circledR}$, SmartOptics, Bochum, Germany). Digital model of the intraoral scanner (DM) , digital model of the conventional gypsum cast (CM) and those of the extracted natural teeth (NT) were imported to a reverse engineering software (3-matic ${ }^{\oplus}$, Materialise, Leuven, Belgium) in which the three models were registered then DM and CM were compared to their corresponding teeth in NT by distance map calculations.

Results: DM had statistically insignificant better trueness when compared to CM for total dataset $(p=0.15)$, statistically insignificant better trueness for CM when mandibular arches analyzed alone ( $p=0.56)$, while a significantly better DM trueness ( $p=0.013$ ) was found when only maxillary arches were compared.

Conclusions: Our results show that digital impression technique is clinically as good as or better than the current reference standard for study models of orthognathic surgery patients.

Keywords: Intraoral scanners, Digital impression, Conventional impression, Trueness

\section{Background}

Conventional impression taking for dental cast preparation is still the clinical reference standard for replicating the intraoral situation [1]. Yet, such conventional approaches are considered cumbersome, bearing in mind the obstacles and challenges for both patient and dentist, including discomfort, nausea, unsatisfactory taste, time consumption, remakes in case of air bubble inclusion, forceful removal of highly retentive impressions with a risk for potential damage [2].

\footnotetext{
* Correspondence: reinhilde.jacobs@uzleuven.be

'OMFS IMPATH research group, Department of Imaging and Pathology,

Faculty of Medicine, University of Leuven and Oral \& Maxillofacial Surgery, University Hospitals Leuven, Leuven, Belgium

${ }^{4}$ Department of Dental Medicine, Karolinska Institutet, Stockholm, Sweden Full list of author information is available at the end of the article
}

To overcome the drawbacks of conventional methods in dentistry, digital virtual models were introduced by Computer Aided Design/Computer Aided Manufacturing (CAD/CAM) solutions [3, 4]. Digital models can be created by indirect or direct approaches where indirect method uses laser optical scanning or computed tomography imaging of conventional impressions or plaster cast to produce digital virtual models $[5,6]$. The direct method uses an intraoral scanning device to capture the patient dentition directly to produce a digital model that can be used to create temporary or final restorations [7-9].

During the last decade the use of intraoral digital impression systems have been steadily increasing. The possibilities and potential of digital impression taking as compared to the conventional approach may be related to its three dimensional representation on the computer,

(c) The Author(s). 2018 Open Access This article is distributed under the terms of the Creative Commons Attribution 4.0 International License (http://creativecommons.org/licenses/by/4.0/), which permits unrestricted use, distribution, and 
enable its versatile use for diagnostic model fabrication and integrated treatment planning. For clinical use, it is important to gain some idea on time and cost efficiency. These factors were assessed in some studies with a rather promising outcome [1,2]. Another point is the system accuracy. Accuracy assessment has been targeted by several studies [10-13]. Yet, it is important to mention that accuracy is most often determined by standardized quality control measures using an in vitro set-up for assessment of precision and trueness. Precision expresses the closeness of repeated measurements to each other. Trueness describes the deviation of the measurement from the dimensions of a reference object. A higher precision means a more predictable measurement, while high trueness means less deviation from the reference object dimensions [9]. So far most accuracy studies mention low error levels, obtained via in vitro methodological set-ups. However, it should be indicated that all those studies start from laboratory testing or an in vitro model approach. A crucial point may however be the performance and accuracy in the clinic, as compared to the reference standard being conventional impression taking. There are a number of potential advantages, favouring digital impression taking to be implemented in daily dental practice. It is therefore crucial to also obtain information on the accuracy of such systems during in vivo use. It is indeed important to evaluate precision and trueness in a clinical environment in the presence of the patient, the operator and related factors that might affect accuracy such as blood and saliva in the mouth, patient movement, operator movement, obstructions by cheek and tongue, reflection of light by intraoral structures and restrictions of space inside the patient's mouth [14]. Studying precision in the clinical set up can be done by repeating the scan of the same dentition intraorally multiple times and measure the deviations among these impressions [12, 15]. Assessing clinical trueness is more challenging, since the dimensions of the natural dental structure for which the digital or conventional impressions are made need to be captured accurately to be used as a reference model for comparison [16].

Focusing on study models made for orthognathic surgery patients, the aim of this study was to introduce a new method to validate the trueness of digital impressions of teeth scanned with an intraoral scanner and conventional impression (clinical standard) when compared to the corresponding natural teeth after extraction and scanned with a high resolution scanner (gold standard) in an in vivo clinical set-up.

\section{Methods}

\section{Patient selection}

Two patients who required full teeth extraction from Oral and Maxillofacial Surgery department (University
Hospitals of Leuven, Belgium) were included in this study which was approved by the Ethical Review Board of the University Hospitals Leuven (S55619 ML9535, University Hospitals Leuven), signed informed consents were obtained from participants.

\section{Impressions and collecting teeth protocols}

Before the surgical procedure, the upper and lower dentitions of each patient were digitally captured in the dental office with a chair-side intraoral scanner $\left(\operatorname{Trios}^{\circ} 3\right.$ Cart wired, 3Shape, Copenhagen, Denmark) one time by a single experienced operator (first author) according to the manufacturer instructions for full arch scanning. For the mandible, one continuous motion starting on occlusal surfaces of posterior teeth starting from one side to the other with alternating movement on the anterior buccolingual area. Followed by scanning the lingual surfaces of all teeth from one side to the other. Finally rolling to buccal side and scan buccal surfaces of all teeth. For the maxilla, the same procedure was repeated except scanning the buccal surfaces of teeth before palatal ones and ending with scanning the palate.. A digital model (DM) was created and exported in stereolithographic (STL) format for each jaw.

One upper and one lower Conventional alginate (Cavex impressional ${ }^{\circ}$, Cavex, Holland) impressions in best fitting trays were made for each patient by the same operator and sent to the dental lab to create plaster casts. These plaster casts were digitized via high resolution optical scanner (Activity $885^{\circ}$, SmartOptics, Bochum, Germany), with an accuracy of $4 \mu \mathrm{m}$ as provided by the manufacturer. Data was exported in STL format and referred to as conventional model (CM).

During surgery, 30 extracted teeth which had full anatomic crowns or minor defects not affecting the dimensions of the crowns were collected from the patients to be used as reference models. All teeth with major defects or loose were excluded. After cleaning from blood and soft tissue residues, each tooth crown was scanned separately by the same lab scanner Activity $885^{\circ}$. Each tooth was fixed during scanning using a custom made gypsum base with a hole in the middle filled with modeling wax (Fig. 1a) by inserting the root into the wax and keeping the crown clear for scanning (Fig. 1b). Data were exported as natural teeth (NT) in STL format.

\section{Evaluation protocol}

All the STL files of the DM, CM and NT for each patient were imported into a reverse engineering software (3-matic ${ }^{\circ}$, Materialise, Leuven, Belgium) to evaluate the trueness of DM and CM to its corresponding NT model. The steps are summarized in the flow chart in Fig. 2 and described as follows: 


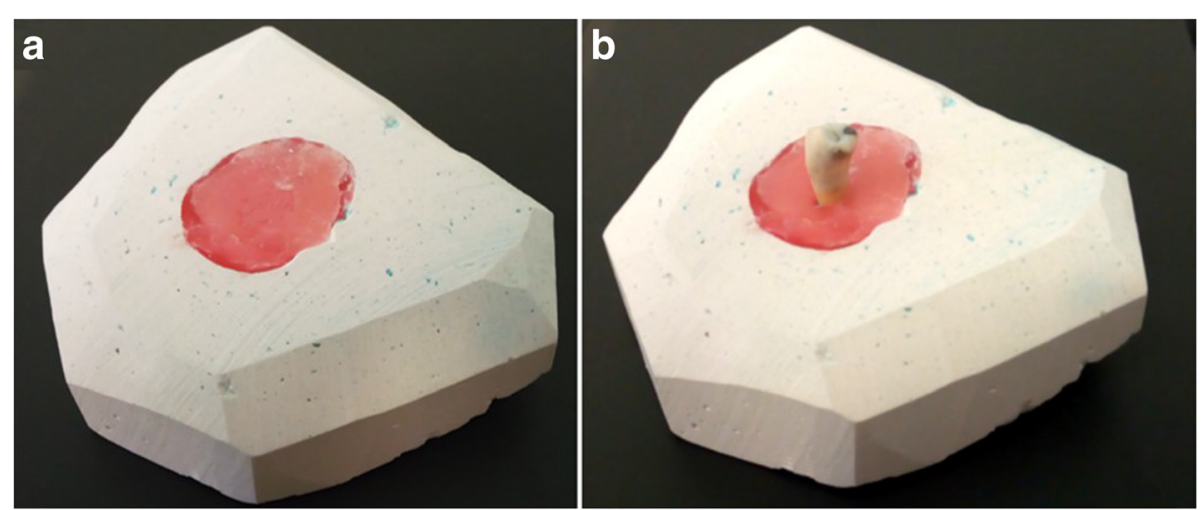

Fig. 1 a Custom made gypsum base used to fix the teeth during scanning. b Each tooth was fixed by inserting its root into wax to keep the crown clear for scanning

(1) Register CM on DM using surface based registration (best fit alignment method).

(2) Register each NT model onto its corresponding DM using surface based registration.

(3) Isolate each tooth group using cutting planes which are parallel to the teeth.

(4) Remove soft tissue from isolated CM and DM models due to lack of soft tissue in NT and to guarantee matched equal borders.

(5) Calculate the distance maps (Euclidean distances) between surfaces using unsigned part comparison in 3-matic software providing a color-coded map. These distance maps were calculated between CM NT and DM - NT separately provided that NT was used as the reference gold standard model in both cases.

The Root Mean Square deviation (RMS) was used to quantify and report degree of conformity of $\mathrm{CM}$ and DM compared to NT. RMS is a frequently used measure of the individual difference between values of a model and the values observed from the original object being modelled. RMS aggregates these individual differences into a single measure of predictive power.

\section{Statistical analysis}

Statistical analysis was done using a dedicated statistical software (MedCalc version 16.4\%, Ostend, Belgium). Normality of distribution was tested by the Shapiro-Wilk
Normality test for CM and DM (maxilla, mandible and both jaws). Non-parametric Wilcoxon matched pairs test was used to compare degree of trueness between CM and DM to NT for maxillary, mandibular and both jaws, level of significance was set at $P<0.05$.

\section{Results}

Table 1 shows median, inter quartile range (IQR), mean and standard deviation (STD) for RMS values for CM and DM when compared with NT.

CM exhibited a total (maxilla and mandible) mean discrepancy and deviation of $133 \pm 45 \mu \mathrm{m}$ (range 10 $-250 \mu \mathrm{m}$ ), while DM showed a total mean discrepancy of $119 \pm 48 \mu \mathrm{m}$ (range $60-280 \mu \mathrm{m}$ ). Figure 3 shows a boxplot for deviations of CM and DM for both arches.

Results of Wilcoxon Matched Pairs Test for comparing $\mathrm{CM}$ and $\mathrm{DM}$ are reported in Table 2, statistically insignificant better trueness for DM compared to CM $(P=0.15)$ was found when total dataset was analyzed, statistically insignificant better trueness for $\mathrm{CM}$ when mandibular arches analyzed alone $(p=0.56)$. While a statistically significant difference was found between DM and CM for maxillary arches alone, favoring DM $(P=0.013)$.

Visual assessment of color-coded deviation maps for teeth showed maximum positive deviations concentrated mainly in two areas: cervical and proximal regions in both CM and DM (Fig. 4), and occlusal surface only in CM (Fig. 5).

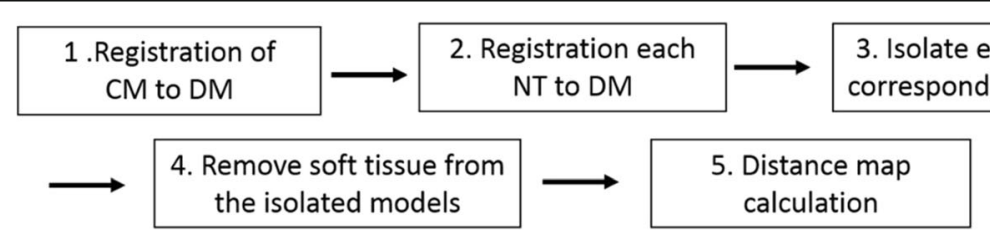

Fig. 2 Evaluation protocol flaw chart 
Table 1 Descriptive statistics of RMS values for CM and DM compared to NT

\begin{tabular}{|c|c|c|c|c|c|c|}
\hline Model & Arch & $\begin{array}{l}\text { Teeth } \\
(\mathrm{N})\end{array}$ & $\begin{array}{l}\text { Median } \\
\mu \mathrm{m}\end{array}$ & $\begin{array}{l}\text { Inter quartile range } \\
\mu \mathrm{m}\end{array}$ & $\begin{array}{l}\text { Mean } \\
\mu \mathrm{m}\end{array}$ & $\begin{array}{l}\text { Standard deviation } \\
\mu \mathrm{m}\end{array}$ \\
\hline \multirow[t]{3}{*}{ CM } & Maxilla & 14 & 151 & 70 & 154 & 45 \\
\hline & Mandible & 16 & 121 & 45 & 120 & 45 \\
\hline & Total & 30 & 130 & 68 & 133 & 45 \\
\hline \multirow[t]{3}{*}{ DM } & Maxilla & 14 & 113 & 65 & 106 & 37 \\
\hline & Mandible & 16 & 118 & 53 & 133 & 57 \\
\hline & Total & 30 & 118 & 38 & 119 & 48 \\
\hline
\end{tabular}

\section{Discussion}

In this study, we presented a new in vivo method to evaluate the trueness of intraoral scanner by comparing conventional and digital impressions to the corresponding natural teeth after extraction.

DM using the former intraoral scanner showed better trueness values $(119 \mu \mathrm{m})$ than $\mathrm{CM}(133 \mu \mathrm{m})$ when both arches were compared. Maxillary arches were significantly higher for DM than CM, while mandibular arches showed insignificant deviations between both methods with higher trueness for $\mathrm{CM}$.

Cervical (buccal, lingual or proximal) deviation areas for $\mathrm{CM}$ can be caused by variable thickness or deficiency of impression materials between neighboring teeth, especially for alginate which has low tear resistance affecting the accuracy of gypsum model (Fig. 4 left) [2, 17-20]. While in DM, this can be caused by light reflection of saliva in interproximal areas as well as the difficulty of scanner light to penetrate these areas (Fig. 4 right) [21] affecting their accuracy in the mandible were saliva has higher concentrations. Another possible effect would be the proximity to gingival tissues which has different light

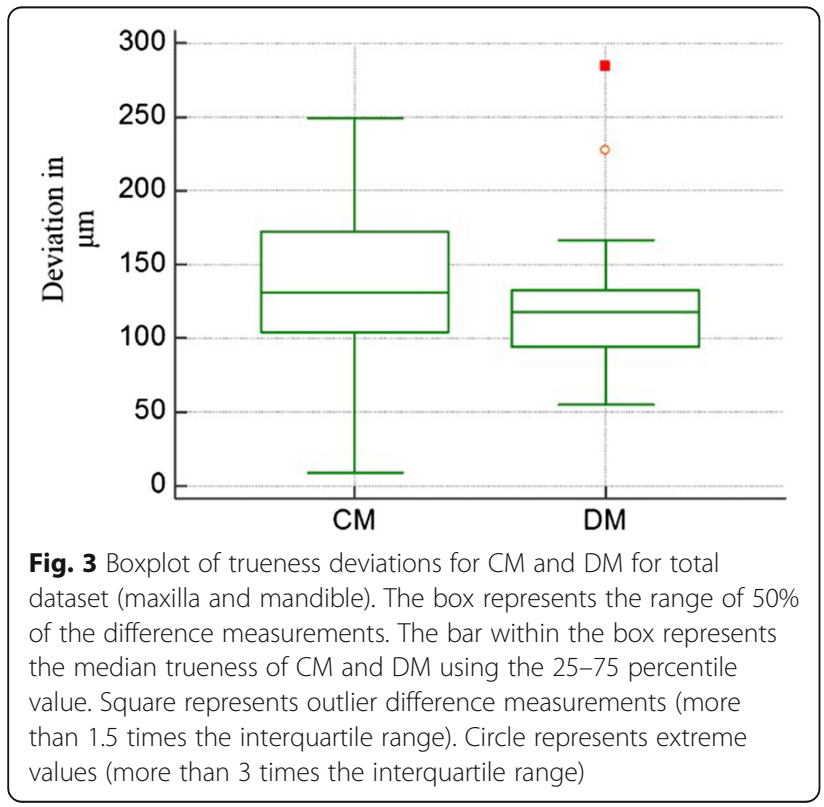

reflective capabilities than tooth structure which might cause potential distortion of light transmitted by intraoral scanner in these areas. Anna et al. [22] found the same area to be affected, it was mentioned that best fit method used for comparison would cause an axial movement of the point-cloud for the model being compared, and such adjustment would cause positive distortions in the margin with reduced distortions at occlusal surface.

The second area with deviations was only shown in $\mathrm{CM}$ on the occlusal surfaces of posterior teeth and palatal (lingual) on anterior teeth. No specific pattern of distribution was detected. This might be attributed to unavoidable trapped air bubbles on the surface of alginate impression which are duplicated on gypsum cast surface and appear as local deviations (Fig. 5 left).

Less saliva content in upper arches, more flexibility and space for scanner tip movement and less movement of maxillary arches compared to mandibular ones could explain significantly better trueness for DM in maxillary arches.

Trueness of intraoral scanners has been examined in few studies, some by taking digital or conventional impressions for industrial manufactured models in controlled lab set-up [23], where the absence of in vivo set-up with its intraoral factors mentioned earlier might affect reported trueness results. Other studies used conventional and digital impressions taken for patients to be compared together using one of them as a reference [24]. These studies neglected the presence of inherited errors in this reference impression or the model created from it leading to less trueness and trusted results. On the other hand, there are studies that used marginal and internal fit of final crown and bridge restorations constructed using digital and conventional impressions to

Table 2 Trueness level of CM and DM to NT

\begin{tabular}{llll}
\hline Model & Arch & N(Teeth) & P-level \\
\hline CM - DM & Maxilla & 14 & $0.013^{*}$ \\
& Mandible & 16 & 0.56 \\
& Total & 30 & 0.15 \\
\hline
\end{tabular}

*indicates significant difference $(P<0.05)$ 

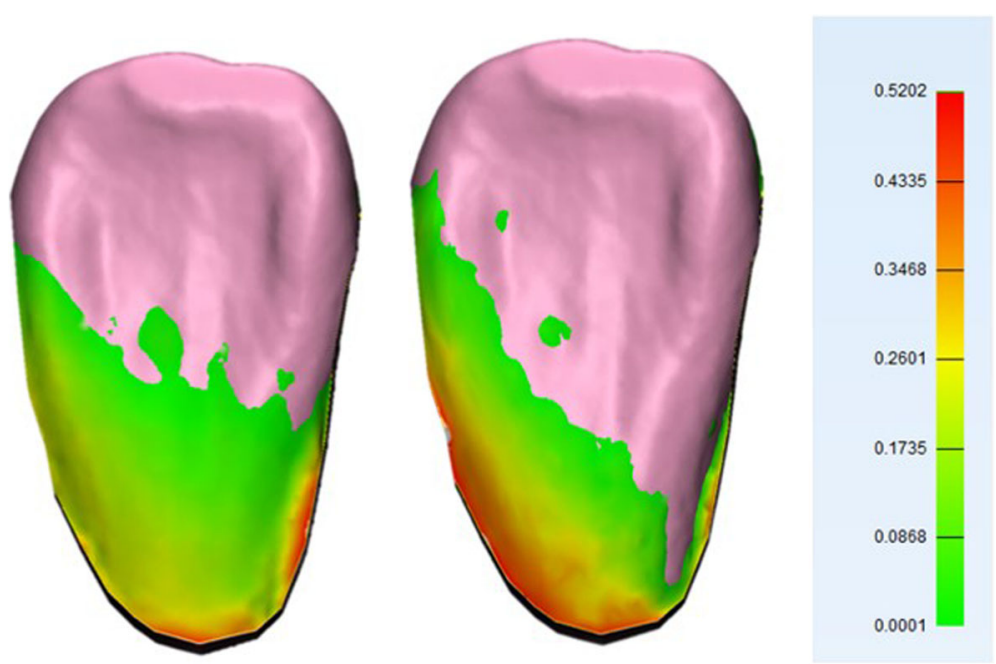

Fig. 4 Color deviation map shows positive deviations in proximal and cervical areas. CM (left), DM (right). A positive value (red) in the color deviation map indicates that the CM and DM are larger than NT in these specific areas

assess the trueness level [21]. In fact, this method involved the trueness of all steps in manufacturing final restoration, and not only the trueness level of impression itself [25].

Intraoral scanners have many advantages which include and not limited to better accuracy values as shown in our results, easy to use, patients' comfort, physical storage space not needed, impressions can be sent directly to milling machine to finalize restorations in minutes, reduced labor work and fast with cost efficient implementation [26, 27], which encourage replacement of conventional impressions by digital ones for many cases of surgical planning and splints manufacturing in maxillofacial surgeries and orthodontic treatments.

In our study, all factors affecting trueness values were neutralized, up to the authors knowledge it was not mentioned in literature that digital and conventional impressions where made in vivo and compared to the same original intraoral structures captured in these impressions. Evaluation was limited to teeth as soft tissue could not be cropped with those teeth (NT) for comparison. Moreover, each tooth was separately evaluated as it is impossible to extract all the teeth and preserve their position accurately on a gypsum base as they are in the patient mouth to be examined for trueness as complete arch.

The use of only one intraoral scanner was another limitation of this study. However, this Trios scanner allows exporting STL files that are compatible with multiple CAD/CAM software. In addition, it supports a powder free, colored scans, ultrafast optical sectioning technique and confocal microscopy scanning technology
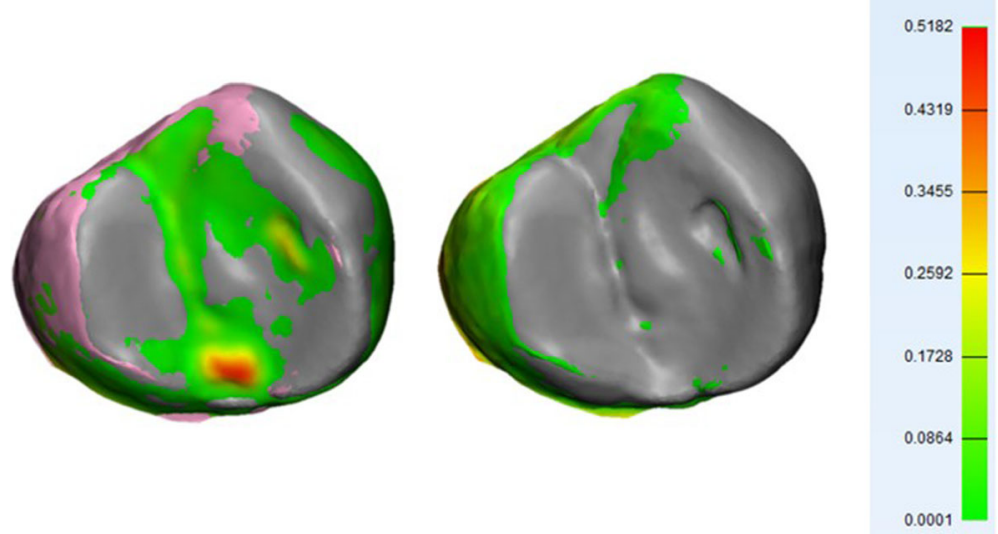

Fig. 5 Positive localized deviations on the surface of CM (left) which are absent on the same surface in DM (right). A positive value (red) in the color deviation map indicates that CM is larger than NT in these specific areas 
$[6,28,29]$. Moreover, this study focused on the introduction of a new method to evaluate the accuracy of intraoral scanners in terms of trueness and not to compare several scanners.

Nevertheless the current results show that the digital impressions technique is clinically as good as or better than the current reference standard used for orthognathic surgery patients. Optimally clinical and digital impression taking for prosthetic work should yield error values of around $25 \mu \mathrm{m}$ [30].

It is recommended to conduct more studies using other types of impression materials and commercial intraoral scanners and include more patient data to report on clinical accuracy rather than technical factory accuracy.

\section{Conclusions}

Direct digital impression methods using intraoral scanning have many advantages over conventional impressions with accurate in vivo results. Yet the present clinical study indicates that the variability in scanner output is still large, with error levels somewhat around or below the conventional impression method.

\section{Abbreviations \\ CAD/CAM: Computer Aided Design/Computer Aided Manufacturing; CM: Conventional gypsum cast model; DM: Digital model of the intraoral scanner; IQR: Inter quartile range; NT: Natural extracted teeth model; RMS: Root Mean Square deviation; STD: Mean and standard deviation; STL: Standard Tessellation or Stereolithographic File}

\section{Funding}

The present study was self-funded, and it was not supported by any grant; therefore, the authors have no conflict of interest related to the present work

\section{Availability of data and materials}

Data are available from the corresponding author, upon request.

\section{Authors' contributions}

All authors made substantial contributions to the present study. EAA, ES, MV and RJ contributed to conception and design, acquisition of data, analysis and interpretation of data; they were, moreover, involved in writing and editing the manuscript. Together, EAA, ES, CP and RJ were the major contributors in preparing and writing the manuscript. FGM and RJ revised the manuscript before submission. All authors read and approved the final manuscript.

\section{Ethics approval and consent to participate}

The present study was approved by the Ethical Review Board of the University Hospitals Leuven (S55619 ML9535, University Hospitals Leuven). Signed informed consents were obtained from participants.

\section{Consent for publication}

Not applicable.

\section{Competing interests}

The authors declare that they have no competing interests in relation to the present work. Francesco Mangano is a Section Editor for BMC Oral Health.

\section{Publisher's Note}

Springer Nature remains neutral with regard to jurisdictional claims in published maps and institutional affiliations.

\section{Author details}

OMFS IMPATH research group, Department of Imaging and Pathology, Faculty of Medicine, University of Leuven and Oral \& Maxillofacial Surgery, University Hospitals Leuven, Leuven, Belgium. ${ }^{2}$ Department of Prosthodontics, Royal Medical Services, Jordanian Armed Forces, Amman, Jordan. ${ }^{3}$ Department of Medicine and Surgery, Dental School, University of Varese, Varese, Italy. ${ }^{4}$ Department of Dental Medicine, Karolinska Institutet, Stockholm, Sweden.

Received: 19 April 2018 Accepted: 21 June 2018

Published online: 03 July 2018

\section{References}

1. Gjelvold B, Chrcanovic BR, Korduner EK, Collin-Bagewitz I, Kisch J. Intraoral digital impression technique compared to conventional impression technique. A randomized clinical trial. J Prosthodont. 2016;25(4):282-7.

2. Patzelt SBM, Lamprinos $C$, Stampf $S$, Att W. The time efficiency of intraoral scanners. An in vitro comparative study J Am Dent Assoc. 2014;145(6):542-51.

3. Otto T, De Nisco S. Computer-aided direct ceramic restorations: a 10-year prospective clinical study of Cerec CAD/CAM inlays and onlays. Int J Prosthodont. 2002:15(2):122-8.

4. Mörmann WH. The evolution of the CEREC system. J Am Dent Assoc. 2006; 137 Suppl(September):7S-13S.

5. Güth JF, Keul C, Stimmelmayr M, Beuer F, Edelhoff D. Accuracy of digital models obtained by direct and indirect data capturing. Clin Oral Investig. 2013;17(4):1201-8.

6. Fasbinder DJ. Computerized technology for restorative dentistry. Am J Dent. 2013;26(3):115-20.

7. Beuer F, Schweiger J, Edelhoff D. Digital dentistry: an overview of recent developments for CAD/CAM generated restorations. Br Dent J. 2008;204(9):505-11

8. Mangano F, Gandolfi A, Luongo G, Logozzo S. Intraoral scanners in dentistry: a review of the current literature. BMC Oral Health. 2017;17(1):149.

9. Ender A, Mehl A. Accuracy of complete-arch dental impressions: a new method of measuring trueness and precision. J Prosthet Dent. 2013; 109(2):121-8.

10. Rödiger $M$, Heinitz $A$, Bürgers $R$, Rinke $S$. Fitting accuracy of zirconia single crowns produced via digital and conventional impressions-a clinical comparative study. Clin Oral Investig. 2017;21(2):579-87.

11. Cho SH, Schaefer O, Thompson GA, Guentsch A. Comparison of accuracy and reproducibility of casts made by digital and conventional methods. J Prosthet Dent. 2015;113(4):310-5.

12. Imburgia M, Logozzo S, Hauschild U, Veronesi G, Mangano C, Mangano FG. Accuracy of four intraoral scanners in oral implantology: a comparative in vitro study. BMC Oral Health. 2017;17(1):92.

13. Mangano FG, Veronesi G, Hauschild U, Mijiritsky E, Mangano C. Trueness and precision of four intraoral scanners in oral implantology: a comparative in vitro study. PLoS One. 2016;11 (9):e0163107.

14. Luthardt RG, Walter MH, Weber A, Koch R, Rudolph H. Clinical parameters influencing the accuracy of 1- and 2-stage impressions: a randomized controlled trial. Int J Prosthodont. 2007;21:322-7.

15. Flügge TV, Schlager S, Nelson K, Nahles S, Metzger MC. Precision of intraoral digital dental impressions with iTero and extraoral digitization with the iTero and a model scanner. Am J Orthod Dentofac Orthop. 2013;144(3):471-8.

16. Ender A, Mehl A. Accuracy in dental medicine, a new way to measure trueness and precision. J Vis Exp. 2014;86:e51374.

17. DeLong R, Knorr S, Anderson GC, Hodges J, Pintado MR. Accuracy of contacts calculated from 3D images of occlusal surfaces. J Dent. 2007; 35(6):528-34.

18. Endo T, Finger WJ. Dimensional accuracy of a new polyether impression material. Quintessence Int. 2006;37(1):47-51.

19. Chen SY, Liang WM, Chen FN. Factors affecting the accuracy of elastometric impression materials. J Dent. 2004;32(8):603-9.

20. Ceyhan JA, Johnson GH, Lepe X, Phillips KM. A clinical study comparing the three-dimensional accuracy of a working die generated from two dual-arch trays and a complete-arch custom tray. J Prosthet Dent. 2003;90(3):228-34.

21. Boeddinghaus M, Breloer ES, Rehmann P, Wöstmann B. Accuracy of singletooth restorations based on intraoral digital and conventional impressions in patients. Clin Oral Investig. 2015;19(8):2027-34. 
22. Persson ASK, Odén A, Andersson M, Sandborgh-Englund G. Digitization of simulated clinical dental impressions: virtual three-dimensional analysis of exactness. Dent Mater. 2009;25(7):929-36.

23. Vecsei B, Joós-Kovács G, Borbély J, Hermann P. Comparison of the accuracy of direct and indirect three-dimensional digitizing processes for CAD/CAM systems - an in vitro study. J Prosthodont Res. 2017:61(2):177-84.

24. Gan N, Xiong Y, Jiao T. Accuracy of intraoral digital impressions for whole upper jaws, including full dentitions and palatal soft tissues. PLoS One. 2016; 11(7):1-15.

25. Seelbach P, Brueckel C, Wöstmann B. Accuracy of digital and conventional impression techniques and workflow. Clin Oral Investig. 2013;17(7):1759-64.

26. Patzelt SBM, Vonau S, Stampf S, Att W. Assessing the feasibility and accuracy of digitizing edentulous jaws. J Am Dent Assoc. 2013;144(8):914-20

27. Patzelt SBM, Emmanouilidi A, Stampf S, Strub JR, Att W. Accuracy of full-arch scans using intraoral scanners. Clin Oral Investig. 2014;18(6):1687-94.

28. Hack GD, SBM P. Evaluation of the Accuracy of Six Intraoral Scanning Devices. An in-vitro Investigation. 2015;10(4):1-5.

29. Franceschini G. A comparative analysis of intraoral $3 d$ digital scanners for restorative dentistry. Internet J Med Technol. 2011;5(1):1-18.

30. Hamalian TA, Nasr E, Chidiac JJ. Impression materials in fixed prosthodontics: influence of choice on clinical procedure. J Prosthodont. 2011:20(2):153-60.

Ready to submit your research? Choose BMC and benefit from:

- fast, convenient online submission

- thorough peer review by experienced researchers in your field

- rapid publication on acceptance

- support for research data, including large and complex data types

- gold Open Access which fosters wider collaboration and increased citations

- maximum visibility for your research: over $100 \mathrm{M}$ website views per year 